\title{
Compliant space mechanisms: a new frontier for compliant mechanisms
}

\author{
R. M. Fowler, L. L. Howell, and S. P. Magleby
}

Department of Mechanical Engineering, Brigham Young University, Provo, UT 84602, USA

Received: 1 March 2011 - Revised: 1 August 2011 - Accepted: 25 September 2011 - Published: 20 October 2011

\begin{abstract}
Compliant mechanisms offer distinct advantages for use in space that can address many of the issues encountered with current rigid-link space mechanisms. Compliant space mechanisms are defined as moveable mechanical assemblies that achieve their desired motion, force, or displacement by means of the deflection of flexible members and can perform a necessary function in the environments of launch and space. Many current space mechanisms are already highly optimized, yet they still experience inherent challenges, and it is unclear if significant improvements in performance can be made by continuing to refine current designs. Compliant space mechanisms offer a promising opportunity to change the fundamental approach to achieving controlled motion in space systems and have potential for dramatic increases in mechanism performance given the constraints of the space environment. This paper proposes the merger of the fields of compliant mechanisms and space mechanisms as a future direction of research in compliant mechanisms, discusses in detail the motivation to do so, and addresses the key factors of applying compliant mechanism technology to space mechanisms.
\end{abstract}

\section{Introduction}

Satellites, rovers, the international space station, and other space vehicles require mechanisms to perform mechanical tasks. These space mechanisms have been designed to perform in the demanding environments of space and launch. Efforts are continually being made to improve their performance and reliability while considering cost (Boesiger, 2008). Many current mechanisms are already highly optimized and it is unclear if significant improvements in performance can be made by continuing to refine current designs. Compliant mechanisms offer a promising opportunity to change the fundamental approach to achieving controlled motion in space systems and have potential for dramatic increases in mechanism performance given the constraints of the space environment. Compliant mechanisms gain motion from the elastic deflection of flexible components. Advantages of compliant mechanisms over traditional mechanisms include increased performance through reduced weight, increased precision, reduced friction, elimination of lubricants, ease of miniaturization, and integration of functions into fewer components. Figure 1 shows a few examples

Correspondence to: L. L. Howell (lhowell@byu.edu) of compliant mechanisms designed by the Compliant Mechanisms Research Group (CMR) at Brigham Young University. The field of compliant mechanisms has matured to the point that design and analysis methods, and the increase in commercial compliant mechanisms, makes it possible to apply them in critical applications.

This paper proposes Compliant Space Mechanisms as a new research direction in compliant mechanisms, provides the motivation to do so, shows the application and benefits of this research, and discusses the crucial factors in beginning to understand this field.

\section{Proposed research direction}

A compliant space mechanism is a moveable mechanical assembly that achieves its desired motion, force, or displacement by means of the deflection of flexible members and that can perform a necessary function in the environments of launch and space.

The field of compliant space mechanisms has the potential for significant impact on the performance of space mechanisms because compliant mechanisms offer distinct advantages that can address many of the issues encountered in current rigid-link mechanisms. The advent of design and analysis methods for compliant mechanisms allows for design engineers to accurately model flexible segments and to 

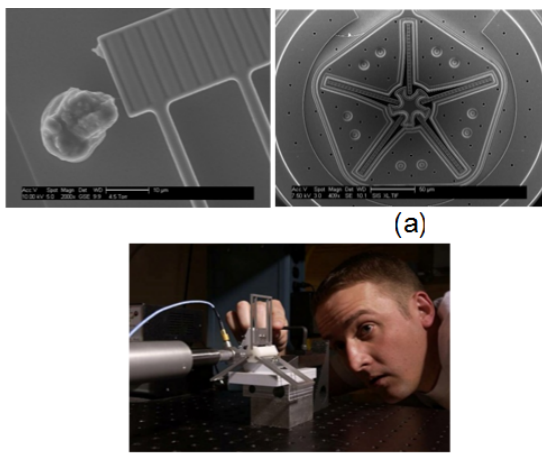

(b)

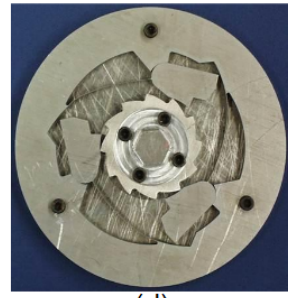

(d)

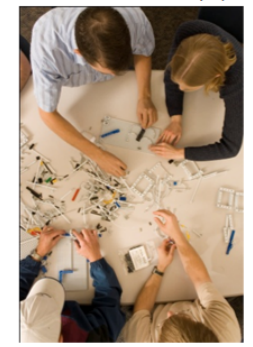

(f) (a)
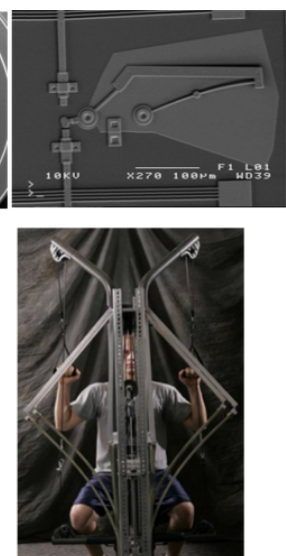

(c)

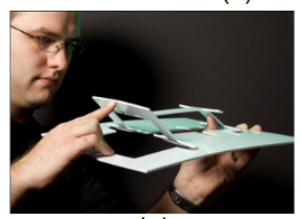

(e)

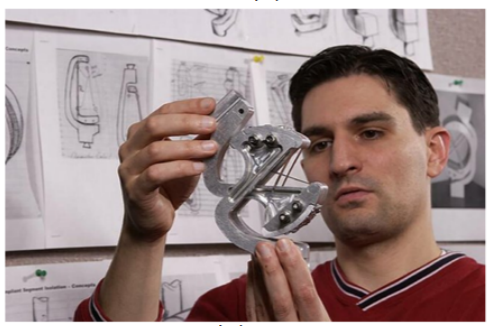

(g)
Figure 1. Examples of compliant mechanisms designed by the CMR: (a) scanning electron micrographs of compliant micro mechanism next to a white blood cell, a micro compliant joint for high g-loads, and a micro bistable mechanism, (b) a high precision device for nanoscribing, (c) an exercise device with specified forcedisplacement characteristics, (d) a compliant overrunning (oneway) clutch, (e) a lamina emergent mechanism (LEM), (f) a custom prototyping kit, compatible with Legos, to facilitate quick design and testing of compliant mechanisms, and (g) a compliant prosthetic knee.

design precision devices for specific tasks. The pseudo-rigidbody model (Howell, 2001) enables flexures to be modeled as rigid-link assemblies with representative link lengths and torsional spring constants. It also enables the use of wellknown rigid-link kinematic and dynamic analysis software packages in analyzing compliant mechanisms. The advent of this approach has brought about many advances in numerous fields and lends itself particularly well to the space industry. Topology optimization (Sigmund, 1997; Frecker et al., 1997; Kim et al., 2006; Saxena and Ananthasuresh, 2000), another method of compliant mechanism design, can be used in the conceptual phase of design to arrive at the optimal geometry for specific loading and boundary conditions. These and other tools, further discussed in Sect. 4.2, provide the ability to merge compliant mechanism technology and space mechanisms so it can be readily used and implemented by designers and analysts in the space industry.

\section{Motivation}

This section addresses the motivation for creating compliant space mechanisms, including the challenges faced by current space mechanisms, the potential advantages of compliant space mechanisms, and limiting factors. The known challenges inherent in compliant mechanisms are discussed, as are the lessons learned from space mechanism failures. The relationship between compliant mechanism research and NASA's Technology Roadmaps is summarized.

\subsection{State of current space mechanisms}

Current space mechanisms are almost entirely composed of traditional rigid-link assemblies. These mechanisms perform a variety of functions. However, the harsh environments of space impose demanding requirements and rigid-link mechanisms can experience a variety of issues, including lubrication outgassing, friction and binding of joints, and inadequate force or torque margin of safety. The mission objectives or desired mechanism functionality also have demanding requirements and rigid-link mechanisms are naturally prone to issues concerning size, weight, and accuracy of motion. Continual effort is put into improving the performance and reliability of space mechanisms, yet many of the inherent challenges still remain and lead to compromises in performance and reliability. Since mechanisms often perform functions that are singularly vital for mission success, a failure could be catastrophic to the mission. Many of the failures of space mechanisms have been documented (Shapiro et al., 1995; Fusaro, 1999) and occur because of the design tradeoffs and inherent challenges.

\subsection{Possible advantages of compliant mechanisms in space applications}

The application of compliant mechanism technology could prove vital in overcoming some of the difficult challenges that current space mechanisms face when put in the space environment. Table 1 shows key challenges of space mechanisms and which advantages of compliant mechanisms could possibly address each challenge.

These advantages eliminate or reduce many of the disadvantages inherent in rigid-link space mechanisms. Compliant mechanisms also offer an increased number of mechanism designs, joints, and configurations. This provides more options in finding an optimized, low-cost mechanism design. The distributed compliance of some compliant mechanisms could be particularly useful in robotic grasping, sample collection, landing platforms, and rover suspensions. Resulting 
Table 1. Current space mechanism challenges and the advantages of compliant space mechanisms that overcome those challenges.

\begin{tabular}{ll}
\hline Challenges of Current Space Mechanism & Advantages of Compliant Space Mechanisms \\
\hline outgassing of lubrication, friction, wear and binding of joints & elimination of joints requiring lubrication or friction \\
\hline large mass/weight (and accompanying cost) & significant part count reduction; miniaturization possible \\
\hline large size/volume & $\begin{array}{l}\text { significant part count reduction, increased number of pos- } \\
\text { sible joints and design configurations, integration of multi- } \\
\text { ple functions into one mechanism, simpler geometries can } \\
\text { lead to a reduction in material and assemblies needed to } \\
\text { achieve the required motion }\end{array}$
\end{tabular}

incorrect mechanism stiffness (components assumed rigid are not entirely rigid)

complex or costly to manufacture and integrate

feedback noise in attitude control system due to mechanism dynamics

single point failure modes

inadequate force or torque margin of safety

lack of accurate modeling and analysis methods for flexible and large-displacement segments

reduced reliability in off-nominal conditions

thermal gradients cause joint binding or misalignment

backlash, hysteresis, and joint misalignment ability to accurately model and predict joint and mechanism stiffnesses; distributed compliance is more accurate for analysis than lumped compliance and it can be accurately calculated

less expensive manufacturing methods possible (e.g. planar); simpler, more integrated geometries can achieve the required motion; significant part count reduction; little or no assembly required

precision motion provides increased predictability and control over mechanism mode shapes, natural frequencies, and component stiffnesses; can improve isolation from deployment dynamics with distributed compliance

redundancy in actuation and motion possible; elimination of lubrication and friction dependent joints

accurate analysis methods; redundancy in actuation and motion possible

proven design and analysis methods

analysis methods provide increased predictability of behavior in off-nominal conditions; reduced susceptibility to foreign objects during testing and operation

mechanisms constructed of a single continuous material

monolithic (single piece) nature of compliant joints eliminates backlash, makes hysteresis predictable, and reduces the need for assembly, thus reducing the possibility of joint misalignment from reduced part count and simpler topologies is the possible effect on a program level of fewer schedule delays during design, procurement, or manufacturing.

Overall, the advantages of compliant space mechanisms provide the opportunity to design simpler, more reliable, better performing, and more cost-effective solutions for many space applications.

\subsection{Limiting factors}

\subsubsection{The space environment}

Much is known about the environment of space and the challenges it presents in designing mechanisms (Sellers, 2004;
Sarafin, 1995; Conley, 1998). The basics of understanding the space environment include the effects of the vacuum, electromagnetic radiation, and charged particles.

The vacuum of space creates three potential problems

- Outgassing: the release of gasses from spacecraft materials, often seen in composites and joint lubrication.

- Cold welding: fusing together of metal components since the vacuum eliminates small air gaps.

- Limited means of heat transfer: heat can only be transfered by radiation between components and space, and by conduction between components in contact. 
The effects of these three problems can be mitigated by employing compliant mechanisms.

Outgassing and cold welding can be avoided by designing joints requiring neither lubrication nor contact surfaces. The often monolithic nature of compliant mechanisms lends itself well to designing joints that overcome these challenges.

The limited means of heat transfer is a problem inherent to the space environment. Compliant space mechanisms need to meet the thermal requirements of their specific mission and must be able to handle the thermal loading or be properly isolated from it. The reduction in number of parts and contact surfaces of compliant mechanisms provides increased simplicity in predicting the temperatures and thermal paths in the mechanism. The thermal properties of space vehicles are important and are carefully analyzed and monitored.

The thermal environment is determined by the temperature and radiation interactions of all the components of the space vehicle and the sun or planets. Convection is not a means of heat transfer in space and excess heat must be properly dissipated from critical components using radiation, or conduction into a neighboring component. A monolithic compliant joint has the potential for significantly better heat transport capability than a multi-element ball bearing.

Electromagnetic radiation from the sun heats up surfaces exposed to it. Whether the mechanism is exposed to the sun or in its shadow can significantly change its temperature and thus its material properties. The resulting thermal extremes can reduce the accuracy and reliability of mechanism performance and can cause damage to surfaces and electronic components. The performance of compliant mechanisms designed for use in space applications must not be adversely affected by the extreme range of temperature. The temperature dependent performance of flexures will need to be better understood.

Charged particles are encountered in the space environment and come from different sources such as the solar wind and flares on the sun, galactic cosmic rays from outside the solar system, or charged particles from the Van Allen radiation belts. They can cause spacecraft charging and possible discharges, sputtering, and single event phenomenon (Sellers, 2004).

Research will be vital in evaluating how these challenges affect compliant space mechanisms and how those differ from the challenges affecting current space mechanisms.

\subsubsection{The launch environment}

The launch environment subjects mechanisms to some of the most intense loads and vibrations experienced on a mission. The launch loads often range from 25 to $100 \mathrm{~g}$ 's. The vibration profile is random and can excite many modes. Understanding these loads and the vibrational response of the mechanisms requires extensive analysis and testing. The stresses, deflections, natural frequencies, mode shapes, and other crucial characteristics will need to be found for any compliant space mechanism.

Compliant mechanisms lend themselves well to vibration isolation applications. A satellite and all its appendages have many needs for vibration isolation. Compliant mechanisms offer the potential to design custom vibration isolation management systems. The design and analysis of largedisplacement flexures for space applications is a matter of energy management. The understanding and control of strain energy in compliant mechanisms can be applied to address this critical need. Compliant mechanisms provide the ability to safely manage the vibrations from launch, separation, or deployment events.

\subsubsection{Performance requirements}

The NASA Space Mechanisms Handbook (Fusaro, 1999) identifies distinct measurable quantities as mechanism performance metrics. They are:

- Range of Motion

- Torque (or Force) Margin

- Operating Speed

- Operating Life

- Pointing Accuracy

- Slew (or Scan) Rate

- Deployment Time

- Restow Capability

- Stowage Duration

Sarafin (1995) also has a listing of similar metrics.

\subsubsection{System requirements}

Aside from mechanism performance, a mechanism must be able to be integrated into the other subsystems and become part of a whole space vehicle. These considerations (Fusaro, 1999) affect not only the mechanism but the entire space vehicle.

- Weight

- Stiffness

- Envelope

- Clearance

- Alignment

- Interface

- Environments (temperature, vibration, shock, vacuum, transportation, and storage) 
These are often vital factors that influence the design of mechanisms and will become avenues of opportunity for innovation as compliant space mechanism design research advances.

\subsubsection{Possible challenges of compliant mechanisms in space applications}

The numerous advantages of compliant mechanisms also come with some challenges. Two distinct challenges are:

- The coupling of motion and forces in compliant mechanisms creates a more complex design situation

- Off-axis stiffness and motion are possible

Typically, a mechanism is designed so it can achieve a certain motion, then, the forces in the joints and links are determined. In compliant mechanisms, the kinematics and dynamics are coupled.

In current space mechanisms, off-axis stiffnesses are significantly larger than the stiffness in the desired direction/axis and are often neglected. In compliant mechanisms, off-axis stiffness can be lower and could create undesirable parasitic motion.

Other known challenges associated with compliant mechanisms are:

- Designing joints or flexures capable of large deflection without failure due to stress, fatigue, or vibration

- Stress relaxation or creep (e.g. under preload, particularly at elevated temperatures or extended stowage duration)

- Designing thin flexible segments to withstand the vacuum and thermal extremes of space (Sellers, 2004)

- Continuously rotating joints often require a hybrid, or partially compliant design

- Designing complex compliant mechanisms that can be manufactured from a planar (or similar) state

- Stored strain energy (can be an advantage or drawback depending on the application)

Fortunately, these challenges are surmountable if proper attention is given to already established compliant mechanism design and analysis guidelines. It will remain the mechanism designer's responsibility to be vigilant in accounting for these challenges. Some aspects, such as manufacturing and testing, will involve a certain level of difficulty due to the stringent requirements for space applications.

\subsection{Documented failures: Space Mechanisms Lessons} Learned Study

The Space Mechanisms Lessons Learned Study performed by NASA (Shapiro et al., 1995) is an extensive collection of knowledge learned from past space mechanism failures, and is evidence that many of the failures in space mechanisms are due to problems for which compliant space mechanisms may provide solutions. The Lessons Learned Study investigated available literature on mechanism failures as well as the research being performed to eliminate those failures. It included a review of the first 28 Annual Proceedings of the Aerospace Mechanism Symposium, documents on deployable appendages from NASA Goddard, an industrial survey that yielded meaningful anomaly reports from companies in the space industry, and a review of the European literature contributed by the European Space Tribology Lab. The "Needs Analysis" section lists future needs for space mechanisms and will be useful in guiding compliant space mechanism research. It begins by stating, "A review of the information compiled for the Lessons Learned study reveals that bearing and lubrication problems are the most prevalent and, thus, improved technologies are most needed in these areas". It then details the specific needs for each of the three categories: Deployable Appendages, Rotating Systems, and Oscillating Systems.

Compliant mechanisms show potential for overcoming lubrication and friction issues caused by traditional mechanisms with lubricated contact surfaces, e.g. ball bearings, and present an opportunity to meet the needs defined by past experience and summarized in the NASA Lessons Learned Study.

\subsection{A call for future direction: NASA Technology Roadmaps}

The NASA Technology Roadmaps provide specific areas where compliant mechanisms may make an immediate impact and help to show how research in this field contributes to technological goals on a national level. The Roadmaps can be found at: http://www.nasa.gov/offices/oct/ home/roadmaps/.

Technology Roadmaps 12 and 9 are most applicable to compliant space mechanisms. Technology Roadmap 12, "Materials, Structures, Mechanical Systems, and Manufacturing" (Piascik et al., 2010) provides detailed technologies that are priority for research and development in the areas most related to compliant mechanisms. Several of the identified technologies are listed in Table 2.

Compliant mechanisms show great promise in these areas. Technology Roadmap 9 "Entry, Descent, and Landing" (Adler et al., 2010) also provides areas for application of compliant space mechanisms, such as: flexible thermal protection systems for entry; mechanical deployments for attached deployable decelerators for descent; and anchoring, 
Table 2. Technologies Identified for Future Development by NASA Technology Roadmap 12 (Piascik et al., 2010).

\begin{tabular}{|c|c|c|}
\hline Roadmap Section & Subsection & Specific Technologies Identified \\
\hline 12.1 Materials & Flexible Material Systems & $\begin{array}{l}\text { Expandable Habitat; Flexible EDL Mate- } \\
\text { rials; Solar Sail; Shape Morphing Materi- } \\
\text { als; Advanced Expandable Materials }\end{array}$ \\
\hline 12.2 Structures & Lightweight Concepts & $\begin{array}{l}\text { Composite/Inflatable Habitats; Expand- } \\
\text { able Structures (Precision Mirrors and So- } \\
\text { lar/Antenna Arrays); Landers }\end{array}$ \\
\hline 12.2 Structures & $\begin{array}{l}\text { Innovative, Multifunctional } \\
\text { Concepts }\end{array}$ & Reusable Modular Components \\
\hline 12.3 Mechanical Systems & $\begin{array}{l}\text { Deployables, Docking and } \\
\text { Interfaces }\end{array}$ & $\begin{array}{l}\text { Common Universal Interchangeable In- } \\
\text { terfaces; Restraint/Release Devices; De- } \\
\text { ployment of Flex Materials; Large } \\
\text { Lightweight Stiff Deployable; Precision } \\
\text { Structure Deploy Mechanism }\end{array}$ \\
\hline 12.3 Mechanical Systems & $\begin{array}{l}\text { Electro-mechanical, } \\
\text { Mechanical and } \\
\text { Micromechanisms }\end{array}$ & $\begin{array}{l}\text { Active Landing Attenuation System; } \\
\text { New Concepts }\end{array}$ \\
\hline 12.3 Mechanical Systems & $\begin{array}{l}\text { Design and Analysis Tools } \\
\text { and Methods }\end{array}$ & Kinematics and Rotor Dynamics Analysis \\
\hline
\end{tabular}

touchdown, and extreme terrain suspension systems for landing.

Some of the areas listed are large scope goals for the future and it is understood that compliant mechanisms would be an integral part of the solutions.

\subsection{Building upon, not replacing, heritage}

From the Russian's Sputnik I (Fig. 2), to present day, an impressive amount has been learned about space mechanisms. Many mechanisms have performed vital functions, such as the sample collection mechanism on the Phoenix Mars Lander (Fig. 3) that uncovered ice on Mars.

This rich heritage is not to be replaced or forgotten; it is to be continued and built upon to take space mechanisms to the next level of performance and reliability.

\section{Merging compliant mechanisms and space mechanisms}

The merger of compliant mechanism technology to space mechanisms is addressed in this section by discussing the current state of space mechanisms, the current state of compliant mechanism research, and the proposed merger of these two fields. Possible technologies to develop and suggested areas to begin research are summarized.

\subsection{Current space mechanisms}

Many earth-sensing satellites, planetary rovers and orbiters, and manned spacecraft have been developed that require mechanisms to perform specific tasks, such as deployments, instrument pointing, stage separations, dockings, sample return, landings, retention and release, attitude stability, etc.

\subsubsection{Space mechanism design rules}

Since the general paradigm for designing space mechanisms has remained fairly consistent over the years, design rules exist for space mechanisms and have been generally well established by industry. A good example of a list of design rules is shown on page 499 in Space Vehicle Mechanisms Elements of Successful Design (Conley, 1998). The NASA Space Mechanisms Handbook (Fusaro, 1999) is the space industry's authoritative document on space mechanism design and contains guidelines and details for space mechanisms of all types. It is available for free to approved US citizens at www.grc.nasa.gov/WWW/spacemech/ (with updates through 2009). The AIAA has a similar document that standardizes how to, for example, calculate margins of safety for a space mechanism (AIAA, 2005) and is commonly used in the space industry. Research needs to be performed in collaboration with these knowledge standards.

In addition to design rules, NASA's Space Mechanisms Handbook (Fusaro, 1999) cites general design objectives for space mechanisms: 


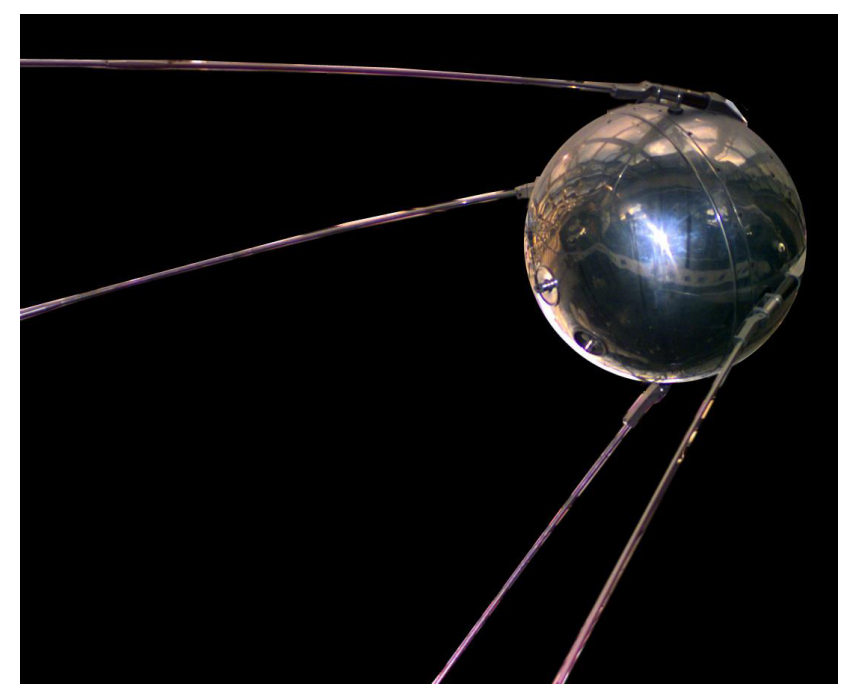

Figure 2. Sputnik I. Courtesy of NASA.

- Identify and eliminate failure modes

- Increase mechanism robustness (performance in off-nominal conditions)

- Provide adequate force or torque margin

- Provide redundancy

- Design for producibility/manufacturability (without compromising performance)

- Design mechanisms for test and analysis

- Provide access for repair

- Provide adequate instrumentation

- Design for storage (stress relaxation, lubrication evaporation)

Many of these design objectives can be met by the advantages of compliant space mechanisms (Table 1).

\subsubsection{Space mechanism types and mechanism components}

Table 3 lists different types of mechanisms needed in space, most of which are identified in the NASA Space Mechanisms Handbook (Fusaro, 1999).

Common deployable mechanisms include: hinges, booms, antennas, covers, solar arrays, trusses, and dampers. Common rotating mechanisms include control moment gyroscopes, momentum wheels, reaction wheels, slip rings, and solar array drives. Common pointing/oscillating mechanisms include gimbals, harmonic drives, bearings, swash plates, pivot joints, antennas, telescopes, and scanning or pointing

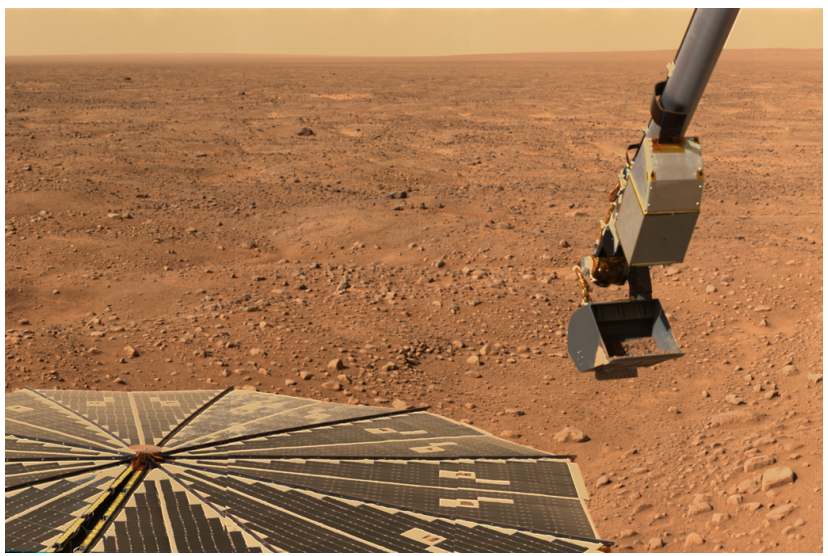

Figure 3. Example of a current space mechanism. Phoenix Mars Lander sample collection shovel. Courtesy of NASA/JPL-CalTechUniversity of Arizona.

Table 3. Space Mechanism Types.

\begin{tabular}{lll}
\hline \multicolumn{3}{c}{ Space Mechanism Types } \\
\hline Deployable & Rotating & Suspension \\
Restraint and Release & Latches and Stops & Drive \\
Vibration Isolation & Separation & Landing \\
Sample Return & Docking & Shape Control \\
Pointing/Oscillating & & \\
\hline
\end{tabular}

mirrors. Precision pointing mirrors are common in space astronomy and can often have tight requirements for pointing, even needing to have a resolution of 1 microradian. A common example of a restraint and release mechanism is a pyrotechnic separation nut used to disconnect the solar arrays from the structure at deployment. A common example of a separation mechanism is known as a Lightband, which releases the payload from the launch vehicle at the separation stage. An example of a mobility suspension mechanism is the Mars Rover rocker-bogie suspension or the ATHLETE rover multi-DOF legs.

\subsection{Current state of compliant mechanisms}

Significant work has already been done in the field of compliant mechanisms. Many compliant mechanisms have become commercially available and research is ongoing in many areas of application. A complete review of the research and publications in compliant mechanisms is not feasible to present here. Compliant mechanisms symposia are held annually as part of the ASME Mechanisms \& Robotics Conference, and the International Symposium on Compliant Mechanisms has been held every four years. Significant advances have been made in compliant mechanism:

- analysis and design methods (e.g. the pseudo-rigidbody model (Howell, 2001), topology optimization 
(Sigmund, 1997, Frecker et al., 1997; Kim et al., 2006; Saxena and Ananthasuresh, 2000), and FACT (Hopkins and Culpepper, 2010a,b; Chen and Culpepper, 2006))

- advanced applications (e.g. lamina emergent mechanisms (Jacobsen et al., 2010; Winder et al., 2008; Albrechtsen et al., 2010; Gollnick, 2010), bistable mechanisms (Jensen and Howell, 2003; Howell, 2001; Qiu et al., 2004; Lusk and Howell, 2008a), constant-force mechanisms (Weight, 2001; Howell, 2001), metamorphic mechanisms (Dai and Jones, 2005, 1999; Wuxiang and Xilun, 2009), large-displacement mechanisms (Trease et al., 2005; Howell, 2001), contact-aided mechanisms (Mankame and Ananthasuresh, 2007, 2004; Cannon and Howell, 2005; Halverson et al., 2010; Mehta et al., 2009), spherical mechanisms (Wilding et al., 2011; Lusk and Howell, 2008b), embedded actuators and sensors (Trease and Kota, 2009), and statically balanced mechanisms (Hoetmer et al., 2010, 2009)).

A Handbook of Compliant Mechanisms will be published by John Wiley \& Sons in 2012 and will include a library to illustrate a few hundred examples.

The research in compliant mechanisms and their resulting components and systems are available to address many of the issues related to space mechanisms. An active compliant mechanisms research community exists with applications ranging from microelectromechanical systems to shape morphing wings to spinal implants.

\subsection{Applying compliant mechanism technology to space mechanisms}

The types of space mechanisms listed in Table 3 can benefit from the application of compliant mechanism technology. Design and analysis methods, such as the pseudo-rigid-body model, topology optimization and FACT, allow for the design of joints or linkages using flexible segments of all types of boundary conditions. Simple flexures, four bar mechanisms, spherical linkages, and many other types of kinematic chains can be modeled and designed. Revolute, torsional, spherical, planar, and many other types of joints can be designed. Examples could include a fixed-guided flexure for attaching a thermally expanding-contracting telecom waveguide to the structural panels or a one-piece bistable compliant four-bar deployment hinge.

The different types of current space mechanisms (Fusaro, 1999; Conley, 1998; Sarafin, 1995) (also shown in Table 3) can be evaluated for their potential for being replaced by compliant mechanisms. Some types will be better suited for the merger than others. In doing this, the distinct motions and functions (Shean-Juinn and Kota, 1999; Fusaro, 1999) that are commonly required by space mechanisms need to be defined. Working from the undertstanding of current space mechanism functions and requirements, compliant space mechanisms can be created that are either compliant redesigns of current space mechanisms or are novel mechanism designs that fulfill a required function or motion. This can be done using a toolbox of compliant mechanism joint types (Olsen et al., 2009; Trease et al., 2005; Winder et al., 2008; Howell, 2001) and design techniques (Howell, 2001; Shean-Juinn and Kota, 1999; Jacobsen et al., 2010; Trease et al., 2005; Berglund et al., 2000; Guerinot et al., 2005). Design guidelines and rules need to be developed on how to apply compliant mechanism technology to space mechanisms so that the process is standardized and widely usable. The breadth of possible applications and configurations of compliant mechanisms in space applications should also be explored.

Candidate designs can be evaluated against the design criteria that has been developed. Promising candidate designs will be selected for detailed analysis. Analysis of the mechanisms will be performed to better understand the behavior of the mechanisms, especially in terms of their desired functionality in the space environments. The performance of the mechanisms should be quantified in several categories, which were outlined earlier as the Performance and System Requirements of the NASA Space Mechanisms Handbook or similarly in Conley (1998); Sarafin (1995).

Testing will be performed following the same standards that are used to qualify flight hardware. The testing results will be compared to the analytical results and models can be verified or corrected, and added to the pool of design knowledge. Efforts should be taken to not only evaluate the performance of the mechanism but to optimize its design and performance. The lessons learned through this process of design, analysis, and testing will be documented as initial guidelines for this field and act as a stepping stone for continued advancements.

\subsubsection{Example of a compliant space mechanism}

Although not yet widespread, flexible links have been successfully used on space mechanisms (Conley, 1998) and the field is just beginning to be explored (Gore et al., 2006). Compliant vibration isolation systems such as SoftRide are produced by CSA Engineering (a division of Moog) and have been used on numerous satellite missions. Figures 4 and 5 show these compliant mechanisms being effectively used to isolate a satellite from launch vehicle vibrations. Similar mechanisms have been used on the Hubble Space Telescope for on-orbit jitter reduction and solar array vibration damping. Propellant tank tab flexures and compliant universal joints are other common examples.

\subsubsection{Possible concepts to develop for compliant space mechanisms}

In addition to converting current space mechanisms to compliant mechanisms, innovative compliant mechanism designs 


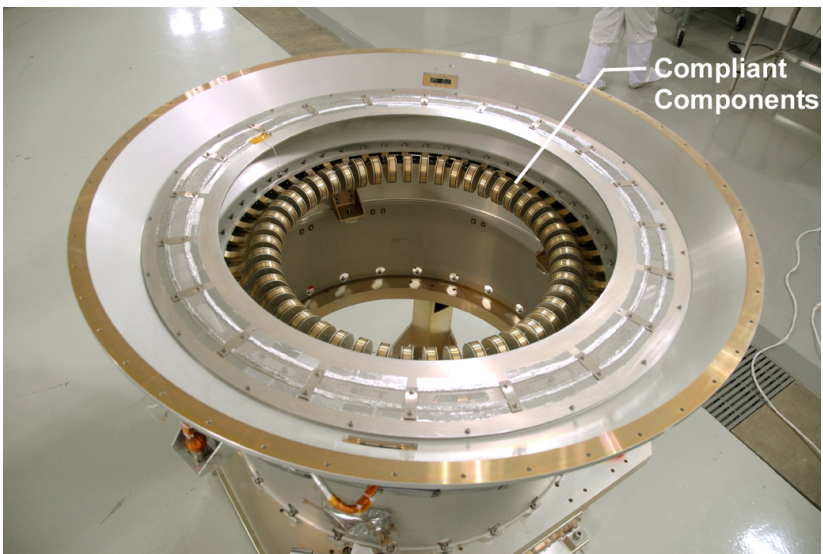

Figure 4. Example of a compliant space mechanism. SoftRide vibration isolation mechanism. Compliant segments are labeled. Courtesy of U.C. Berkeley.

may be developed. This could include the development of the concepts listed here:

- Compliant joints capable of 90 and even 180 degree deflection; multiple segment joints

- Statically balanced hingeline joints

- Compliant joints with improved off-axis properties

- Temperature insensitive joints

- Modular vibration isolation mechanisms

- Compliant bi/tri-stable hinges

- Creasing joints for deployable arrays and masts

- Integrated deployment springs

- Redundant compliant latches

- Multi-Layer lamina emergent thermal radiators

- Pointing array mechanisms

- Two/three axis compliant gimbal platforms

- Compliant joints with integrated damping

\subsection{Areas of research and questions to answer}

Merging the fields of compliant and space mechanisms opens up many possible areas of research. Research can be started in a variety of ways. Each of the space mechanism types should be looked at for their compliant potential. A desired mechanism function and its requirements could be the starting point. Replacing a current mechanism component, such as a ball bearing, would yield valuable results. Designing to

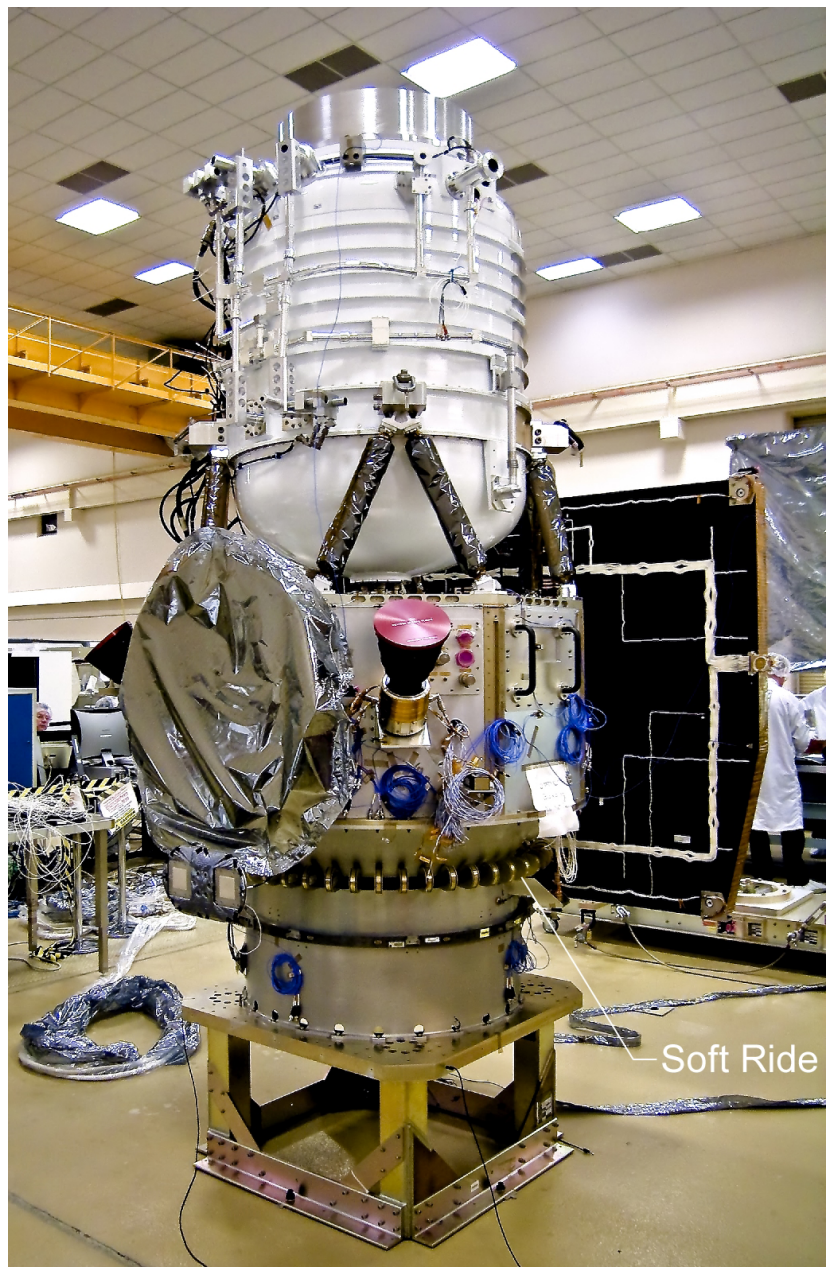

Figure 5. Example of a compliant space mechanism. SoftRide vibration isolation mechanism. The compliant segments separate the launch vehicle adapter and the satellite WISE that was launched on a Delta II in December 2009. Courtesy of U.C. Berkeley.

overcome a reoccurring issue or failure in a current mechanism could guide the research. Guidelines, methods, and tools for the design, analysis, and testing of compliant space mechanisms need to be developed and established for use in industry.

This research hopes to yield ground-breaking answers to detailed questions such as this one: how do compliant segments, especially large-displacement, behave in the thermal extremes and the vacuum of space? There are many fascinating questions and there will be even more as this field unfolds.

Compliant space mechanism research will help fulfill the needs expressed in the NASA Technology Roadmaps and Lessons Learned Study, while also creating next generation space mechanisms. 


\section{Conclusions}

Failures in space can be costly, dangerous, and can hinder progress of technology development and space exploration. The field of compliant space mechanisms has the potential for significant impact on the performance of future space mechanisms. Research in compliant space mechanisms will create innovative new space technologies. It could initiate critical advances that would lead to more affordable and more capable space mechanism solutions. This research cross-cuts several technologies and applies to a large range of different space mechanism types.

Compliant space mechanism research hopes to inspire collaboration between government space agencies (such as NASA), companies in the space industry, and academia. Overall, it would present opportunities for game-changing technologies and lead to enhanced capabilities in space exploration.

Acknowledgements. This material is based upon work supported by the National Science Foundation under Grant No. CMMI-0800606. Any opinions, findings, and conclusions or recommendations expressed in this material are those of the authors and do not necessarily reflect the views of the National Science Foundation.

Edited by: J. Herder

Reviewed by: B. Trease and another anonymous referee

\section{References}

AIAA: AIAA S-114, Moving Mechanical Assemblies for Space and Launch Vehicles, 2005.

Albrechtsen, N. B., Magleby, S. P., and Howell, L. L.: Using Technology Push Product Development to Discover Potential Applications and Categories of Enabled Opportunities for Lamina Emergent Mechanisms, in: ASME 2010 International Design Engineering Technical Conference, DETC2010-28531, ASME, Montreal, Quebec, Canada, 2010.

Adler, M., Wright, M., Campbell, C., Clark, I., Engelund, W., and Rivellini, T.: Entry, Descent, and Landing Roadmap - Technology Area 09, NASA, draft edn., 2010.

Berglund, M. D., Magleby, S. P., and Howell, L. L.: Design Rules for Selecting and Designing Compliant Mechanisms for RigidBody Replacement Synthesis, in: ASME 2000 Design Engineering Technical Conference, DETC00/DAC-14225, ASME, Baltimore, Maryland, 2000.

Boesiger, E. A. (Ed.): 39th Aerospace Mechanisms Symposium, NASA/CP-2008-215252, NASA and Lockheed Martin Space Systems Company, 2008.

Cannon, J. R. and Howell, L. L.: A compliant contact-aided revolute joint, Mech. Mach. Theory, 40, 1273-1293, 2005.

Chen, S.-C. and Culpepper, M. L.: Design of a six-axis micro-scale nanopositioner - HexFlex, Precis. Eng., 30, 314-324, 2006.

Conley, P. L. (Ed.): Space Vehicle Mechanisms - Elements of Successful Design, John Wiley \& Sons, Inc., 1998.
Dai, J. S. and Jones, J. R.: Mobility in metamorphic mechanisms of foldable/erectable kinds, J. Mech. Des.-T. ASME, 121, 375-382, 1999.

Dai, J. S. and Jones, J. R.: Matrix representation of topological changes in metamorphic mechanisms, J. Mech. Des.-T. ASME, 127, 837-840, 2005.

Frecker, M., Ananthasuresh, G., Nishiwaki, S., Kikuchi, N., and Kota, S.: Topological synthesis of compliant mechanisms using multi-criteria optimization, J. Mech. Des.-T. ASME, 119, 238245, 1997.

Fusaro, R. L. (Ed.): NASA Space Mechanisms Handbook, Glenn Research Center NASA/TP 206988, Cleveland, OH, 1999.

Gollnick, P. S.: Design Principles and Preliminary Actuation Approaches for Novel Multiple-Layer Lamina Emergent Mechanisms, Master's thesis, Brigham Young University, 2010.

Gore, B., Didziulis, S., and Hilton, M.: Space Vehicle Mechanisms, Crosslink Magazine (Aerospace Corporation), vol. 12, available at: http://www.aero.org/publications/crosslink/fall2006/03.html, 2006.

Guerinot, A. E., Magleby, S. P., Howell, L. L., and Todd, R. H.: Compliant Joint Design Principles for High Compressive Load Situations, J. Mech. Design, 127, 774-781, 2005.

Halverson, P. A., Howell, L. L., and Magleby, S. P.: Tension-Based Multi-stable Compliant Rolling-contact Elements, Mech. Mach. Theory, 45, 147-156, 2010.

Hoetmer, K., Herder, J., and Kim, C.: A building block approach for the design of statically balanced compliant mechanisms, in: ASME International Design Engineering Technical Conferences and Computers and Information in Engineering Conference, vol. 7, 313-323, ASME, dETC2009, 2009.

Hoetmer, K., Woo, G., Kim, C., and Herder, J.: Negative Stiffness Building Blocks for Statically Balanced Compliant Mechanisms: Design and Testing, Journal of Mechanisms and Robotics, 2, 041007-1-041007-7, 2010.

Hopkins, J. B. and Culpepper, M. L.: Synthesis of multi-degree of freedom, parallel flexure system concepts via Freedom and Constraint Topology (FACT) - Part I: Principles, Precision Engineering, 34, 259-270, 2010a.

Hopkins, J. B. and Culpepper, M. L.: Synthesis of multi-degree of freedom, parallel flexure system concepts via freedom and constraint topology (FACT). Part II: Practice, Precis. Eng., 34, 271278, 2010b.

Howell, L. L.: Compliant Mechanisms, John Wiley \& Sons, Inc., 2001.

Jacobsen, J. O., Winder, B. G., Howell, L. L., and Magleby, S. P.: Lamina Emergent Mechanisms and Their Basic Elements, Journal of Mechanisms and Robotics, 2, 1-9, 2010.

Jensen, B. D. and Howell, L. L.: Identification of Compliant Pseudo-Rigid-Body Four-Link Mechanism Configurations Resulting in Bistable Behavior, J. Mech. Design, 125, 701-708, 2003.

Kim, J. C., Kota, S., and Moon, Y.: An instant center approach toward the conceptual design of compliant mechanisms, J. Mech. Des.-T. ASME, 128, 542-550, 2006.

Lusk, C. P. and Howell, L. L.: Spherical Bistable Micromechanism, J. Mech. Design, 130, 1-6, 2008a.

Lusk, C. P. and Howell, L. L.: Components, Building Blocks, and Demonstrations of Spherical Mechanisms in Microelectromechanical Systems, J. Mech. Design, 130, 034503-1-034503-4, 
2008b.

Mankame, N. D. and Ananthasuresh, G. K.: Topology optimization for synthesis of contact-aided compliant mechanisms using regularized contact modeling, Comput. Struct., 82, 1267-1290, 2004.

Mankame, N. D. and Ananthasuresh, G. K.: Synthesis of contactaided compliant mechanisms for non-smooth path generation, Int. J. Numer. Meth. Eng., 69, 2564-2605, 2007.

Mehta, V., Frecker, M., and Lesieutre, G. A.: Stress relief in contact-aided compliant cellular mechanisms, J. Mech. Des.-T. ASME, 131, 091009-1-091009-11, 2009.

Olsen, B. M., Hopkins, J. B., Howell, L. L., Magleby, S. P., and Culpepper, M. L.: A Proposed Extendable Classification Scheme for Compliant Mechanisms, Proceedings of the ASME International Design Engineering Technical Conferences, San Diego, CA, 30 August-2 September, DETC2009-87290, 2009.

Piascik, B., Vickers, J., Lowry, D., Scotti, S., Stewart, J., and Calomino, A.: Materials, Structures, Mechanical Systems, and Manufacturing Roadmap - Technology Area 12, NASA, draft edn., 2010.

Qiu, J., Lang, J. H., and Slocum, A. H.: A Curved-Beam Bistable Mechanism, J. Microelectromech. S., 13, 137-146, 2004.

Sarafin, T. P. (Ed.): Spacecraft Structures and Mechanisms: From Concept to Launch, Microcosm Press and Springer, 1995.

Saxena, A. and Ananthasuresh, G. K.: On an optimal property of compliant topologies, Struct. Optimization, 19, 36-49, 2000.

Sellers, J. J.: Understanding Space - An Introduction to Astronautics, McGraw Hill Custom Publishing, revised second edn., 2004.
Shapiro, W., Murray, F., and Howarth, R.: NASA Space Mechanisms Lessons Learned Study, vol. 1-Summary, NASA Lewis Research Center Cleveland, Ohio TM 107046, 1995.

Shean-Juinn, C. and Kota, S.: Automated conceptual design of mechanisms, Mech. Mach. Theory, 34, 467-495, 1999.

Sigmund, O.: On the Design of Compliant Mechanisms Using Topology Optimization, Mechanics Based Design of Structures and Machines, MARCEL DEKKER INC, 25, 493-524, 1997.

Trease, B. and Kota, S.: Design of adaptive and controllable compliant systems with embedded actuators and sensors, J. Mech. Des.-T. ASME, 131, 111001-1-111001-12, 2009.

Trease, B. P., Moon, Y.-M., and Kota, S.: Design of LargeDisplacement Compliant Joints, J. Mech. Design, 127, 788-798, 2005.

Weight, B. L.: Development and Design of Constant-Force Mechanisms, Master's thesis, Brigham Young University, 2001.

Wilding, S. E., Howell, L. L., and Magleby, S. P.: Spherical Lamina Emergent Mechanisms, Mech. Mach. Theory, in press, 2011.

Winder, B. G., Magleby, S. P., and Howell, L. L.: A Study of Joints Suitable for Lamina Emergent Mechanisms, in: ASME 2008 International Design Engineering Technical Conference, DETC2008-49914, ASME, Brooklyn, New York, 2008.

Wuxiang, Z. and Xilun, D.: A method for designing metamorphic mechanisms and its application, in: ASME International Conference on Reconfigurable Mechanisms and Robots, London, 2009. 